EPJ Web of Conferences 19, 02005 (2012)

DOI: $10.1051 /$ epjconf/20121902005

(C) Owned by the authors, published by EDP Sciences, 2012

\title{
Using frequency maps to explore the distribution function of the Milky Way stellar halo
}

\author{
M. Valluria \\ Department of Astronomy, University of Michigan, Ann Arbor 48109, USA
}

\begin{abstract}
Resolved surveys of the Milky Way's stellar halo can obtain all 6 phase space coordinates of tens of thousands of individual stars, making it possible to compute their 3-dimensional orbits. When frequency mapping is applied to such orbits they also represent the underlying phase space distribution function since the orbits that the are drawn from [3]. A frequency maps clearly separates out the major types of orbits that constitute the DF and their relative abundances. The structure of the frequency maps, especially the locations of resonant orbits, reflects the formation history and shape of the dark matter potential and its orientation relative to the disk. The application of frequency analysis to cosmological hydrodynamic simulations of disk galaxies shows that the orbital families occupied by halo stars and dark matter particles are very similar, implying that stellar halo orbits can be used to constrain the DF of the dark matter halo, possibly impacting the interpretation of results from direct dark matter detection experiments. An application of these methods to a sample of $\sim 16,000$ Milky Way halo and thick disk stars from the SDSS-SEGUE survey yields a frequency map with strong evidence for resonant trapping of halo stars by the Milky Way disk, in a manner predicted by controlled simulations in which the disk grows adiabatically. The application of frequency analysis methods to current and future phase space data for Milky Way halo stars will provide new insights into the formation history of the different components of the Galaxy and the DF of the halo.
\end{abstract}

\section{INTRODUCTION}

The three fundamental frequencies $\left(\Omega_{1}, \Omega_{2}, \Omega_{3}\right)$ of oscillation of an orbit can be accurately extracted using a Fourier Transform method known as frequency analysis [1], [2]. These orbital frequencies can be used to obtain a complete picture of the properties of individual orbits as well as the entire phase space distribution function (DF) [3]. Frequency analysis can be used to distinguish between regular and chaotic orbits, to classify regular orbits into major orbit families, and to quantify the average shape of an orbit and relate its shape to that of the halo [4]. Resonant orbits are regular orbits that have fewer than 3 linearly independent fundamental frequencies which are related via a integer linear equation such as: $l \Omega_{1}+m \Omega_{2}+n \Omega_{3}=0$, where $(l, m, n)$ are small integers. A frequency map constructed from pairs of frequencies (e.g. $\Omega_{1} / \Omega_{3}$ vs. $\Omega_{2} / \Omega_{3}$ ) can be used to easily identify the most important resonant orbit families, since such orbits populate straight lines on such a map. The strength (or importance) of a resonance can be assessed from the number of orbits associated with it. In this contribution will briefly summarize recent applications of frequency mapping to both controlled and cosmological simulations of disk galaxies and to real stellar orbits derived from phase space coordinates obtained with the SDSS SEGUE survey.

\section{EFFECT OF DARK MATTER HALO SHAPE AND ORIENTATION ON ORBITAL PROPERTIES}

Controlled simulations show that the orbits of halo particles retain a "memory" of the global shape of a triaxial halo relative to the disk even when the halo becomes nearly oblate at small radii due to the

\footnotetext{
ae-mail: mvalluri@umich.edu
}

This is an Open Access article distributed under the terms of the Creative Commons Attribution-Noncommercial License 3.0, which permits unrestricted use, distribution, and reproduction in any noncommercial medium, provided the original work is properly cited. 

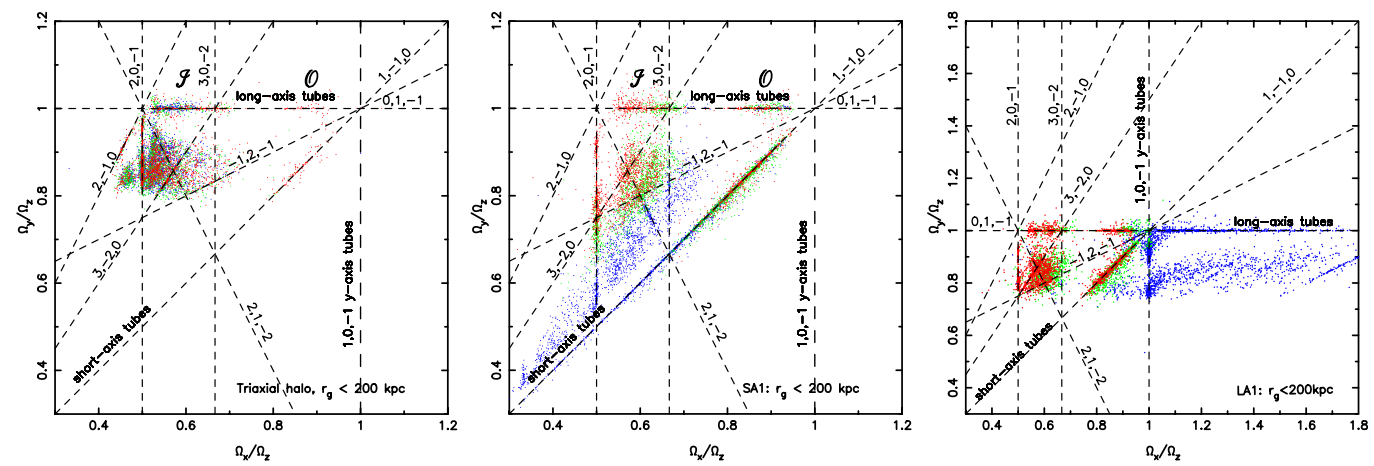

Figure 1. Frequency maps of $\sim 10^{4}$ halo orbits with radius $<200 \mathrm{kpc}$, Left: in a triaxial halo model; Middle: disk perpendicular to halo short-axis (model SA1); Right: disk perpendicular to halo long-axis (model LA1). Dashed lines mark resonances characterized by integers $(l, m, n)$; inner and outer long-axis tubes marked by script I and $\mathrm{O}$ along $\Omega_{y} / \Omega_{z} \sim 1$. Color coding is by energy (blue: tightly bound; green: intermediate; red: weakly bound).

growth of a baryonic component [4]. When ratios of orbital fundamental frequencies $\Omega_{x}, \Omega_{y}, \Omega_{z}$ (in Cartesian coordinates) of a large number $\left(1-2 \times 10^{4}\right)$ halo orbits from a self-consistent $D F$ are plotted in a frequency map (Fig. 1), it represents the orbital content of the DF. Such a map gives an accurate representation of its orbital distribution, even when orbits are selected within the solar neighborhood. In a frequency map different types of orbits clearly separate out into groups and along lines making them easy to identify. For a strongly triaxial halo (created via multiple mergers of spherical NFW halos) the frequency map (Fig. 1[left]) shows numerous points clustered along the horizontal line at $\Omega_{y} / \Omega_{z}=1$ corresponding to long-axis tubes (L-tubes). The clump of points below this line corresponds to nonresonant box orbits and the vertical line at $\Omega_{x} / \Omega_{z}=0.5$ corresponds to resonant boxlet (banana) orbits. Other resonant box orbit families are also populated. We [3] show that when a disk galaxy grows inside such a triaxial halo, in addition to changing the halo's shape, it causes significant resonant trapping of halo orbits. The specific orbit families that are trapped depend strongly on the relative orientation of the disk and the halo. In Figure 1[middle] the disk is perpendicular to the short axis of the original triaxial halo (on left) and therefore traps halo orbits into the short-axis tube (S-tubes) family (seen as the enhanced clustering along diagonal line with $\Omega_{x} / \Omega_{y} \sim 1$ ). In contrast when the disk grows perpendicular to the long-axis of the halo (Fig. 1[right]) it traps orbits into the L-tube family (seen as enhanced clustering along the horizontal line with $\Omega_{y} / \Omega_{z}=1$ ). Thus the relative importance of orbits associated with L-tubes versus S-tubes is reflected by the increased strength of the orbit family that shares the symmetry of the disk and reflects the relative orientation of the disk and halo.

\section{ORBITS OF STARS AND DARK MATTER IN COSMOLOGICAL SIMULATIONS}

We [5] analyzed a simulation of a disk galaxy in a fully cosmological context [6] [part of the McMaster Unbiased Galaxy Simulations sample, hereafter MUGS]. The simulations include low-temperature metal cooling, heating from UV background radiation, star formation, stellar energy, metal feedback, and metal diffusion, and produce fairly realistic disk galaxies. We use frequency analysis to study the orbit populations of both star particles and dark matter particles in the MUGS disk galaxy g15784. For this galaxy the dark matter halo is nearly oblate with flattening parameter $q \sim 0.85$ and the stellar halo is also oblate, but flatter with $q \sim 0.7$ so one would expect S-tubes to dominate the DF. Figure 2 shows histograms of orbits of different types as a function of the orbital pericenter radius $r_{\text {peri }}$. The box orbits are unimportant $(<5 \%)$ for both stars and dark matter particles, and the inner part of the halo is dominated by chaotic orbits ( $42 \%$ of stars, and $38 \%$ of dark matter). At large radii both L-tubes ( $21 \%$ for stars and $28 \%$ for dark matter) and S-tubes (32\% for stars and $29 \%$ for dark matter) are equally 

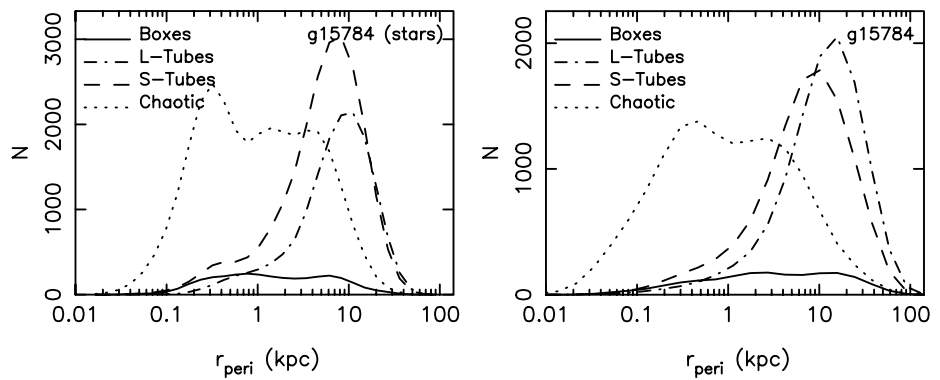

Figure 2. Distribution of orbit types as a function of orbital pericenter distance $r_{\text {peri }}$ for orbits in a cosmological simulation of a disk galaxy (g15784): halo stars (left) and halo dark matter particles (right).

important, contrary to what might be expected simply from the shape of the halo. The similarity between the orbit populations of stars and DM particles is striking. There are subtle differences: more stars than DM are on S-tubes (these stars formed from dissipative mergers); and more DM particles are on L-tubes, indicating that they were accreted with low-angular momentum [4].

\section{EVIDENCE FOR RESONANT TRAPPING OF HALO STARS IN SEGUE DATA}

We [7] have recently analyzed the orbital properties of a subset of stars from the Sloan Digital Sky Survey-SEGUE project [8] whose three dimensional velocities are well-quantified. This set of stars was recently used to show that the stellar halo may consist of two distinct but overlapping structural components with slightly different rotation signatures and metal content [9]. The sample consists of stars with $r-r_{\odot}<4 \mathrm{kpc}$ for which all 6 phase-space coordinates are available and includes thin and thick disk stars but is dominated by halo stars [9]. We used the phase space coordinates to integrate the orbits of these stars in a Milky Way potential modeled by an oblate axisymmetric NFW dark matter halo $(q=0.95)$, a Hernquist bulge, and Miyamoto-Nagai disk with parameters that match the rotation curve of the Milky Way. Since the potential is flattened and axisymmetric, frequency analysis in cylindrical coordinates yields radial, azimuthal and vertical oscillations frequencies $\Omega_{R}, \Omega_{\phi}$ and $\Omega_{z}$. The sign of $\Omega_{\phi}$ is either positive or negative, depending on whether the particle circulates clockwise or anti-clockwise about the Galactic center.

To provide a basis for understanding the frequency map in cylindrical coordinates Fig. 3[left] shows the orbits of 20,000 randomly selected halo particles in a controlled simulation in which a disk galaxy was grown inside a spherical NFW halo with no net rotation [3]. The growth of the disk inside this halo, made it oblate with flattening $q \sim 0.9$ similar to that assumed for our Milky Way model. This controlled adiabatic simulation shows symmetry about $\Omega_{\phi} / \Omega_{R}=0$ because the orbits were selected at random from a DF with no net rotation. The frequency map of 16,000 calibration stars from the SDSS-Segue survey is shown in Fig. 3[middle], while Fig. 3[right] shows a map of halo stars from the MUGS disk galaxy g15784 (see § 3). In the left and middle maps points lie in two distinct lobes, corresponding to orbits with prograde and retrograde motions relative to the disk. In addition they cluster along several thin horizontal lines corresponding to orbital resonances $\Omega_{z} / \Omega_{R}=0.5$ (1:2), 0.66 (2:3), 0.75 (3:4), $0.83(5: 6), 1$ (1:1 and 2:2). Although there are some differences, the similarities in the strengths of the resonances and the specific resonances ratios in the left and middle panels are striking. Figure 3 [right] shows the phase space DF of orbits of stars selected from simulated disk g15784. It is strikingly different from the other two DFs. This galaxy experienced its last major merger $z=2$ but is perturbed even at $z=0$ by scattering of stars by dwarf companions and dark matter subhalos destroys resonances [5]. g15784 also has L-tubes and boxes absent from the other two halos. Although the shapes of the potentials in all three systems are similar (nearly oblate with $(q \sim 0.9)$, the frequency maps readily 

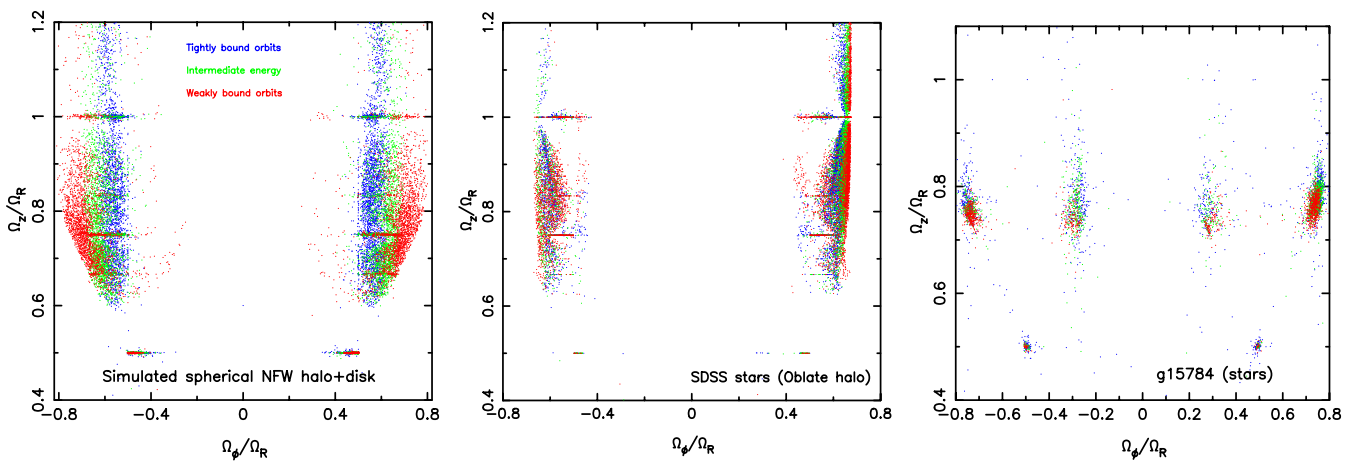

Figure 3. Left: Frequency maps of $10^{4}$ halo orbits in the simulation where a stellar disk grows in a spherical NFW halo. The bisymmetry about $\Omega_{\phi} / \Omega_{R}=0$ results because the simulated halo has no net rotation. The map shows several resonances which appear as horizontal lines: e.g. $\Omega_{z} / \Omega_{R}=1 / 2,2 / 3,3 / 4,5 / 6,1,3 / 2$. Middle: Frequency map of orbits of stars from the SDSS-SEGUE sample evolved in an axisymmetric potential: resonances are seen at $\Omega_{z} / \Omega_{R}=1 / 2,3 / 4,5 / 6,1$. Right: Frequency map of $10^{4}$ halo stars from MUGS galaxy g15784. Only one resonance is seen at $\Omega_{z} / \Omega_{R}=1 / 2$.

reflect similarities and differences in their distribution functions - information that is difficult to uncover via conventional approaches.

\section{CONCLUSIONS}

Previous applications of frequency mapping in Galactic Dynamics have been restricted to uniformly sampled initial conditions designed to study the structure of the underlying potential. When applied to large samples of orbits drawn from a self-consistent distribution function, frequency maps, also represent the phase space distribution function [3]. On the map the major families and resonantly trapped separate out clearly giving clues to the formation process. Similarities between the frequency maps of an adiabatic simulation of a disk galaxy in a spherical halo and orbits derived from SEGUE, suggests that halo stars have been resonantly trapped by the Milky Way's disk. If this result hold up in future surveys, it could be evidence for a relatively quiescent recent history, contradicting predictions from $\Lambda \mathrm{CDM}$. A study of dark matter and stellar orbits in a cosmological hydrodynamical simulation of a disk galaxy shows relatively insignificant differences between these two components suggesting that with large samples of halo orbits, we may be able to gain insights into DF of dark matter particles themselves, significantly impacting future direct dark matter detection searches.

MV is partially supported by NSF grant AST-0908346 and thanks her collaborators particularly J. Bailin, T. Beers, V.P. Debattista, B. O'Shea, T.Quinn and G. Stinson for permission to summarize the results of various applications of frequency mapping, some prior to publication.

\section{References}

[1] Laskar J., Celestial Mechanics and Dynamical Astronomy, 56, (1993), p. 191

[2] Valluri M., \& Merritt D., ApJ, 506, (1998), p. 686

[3] Valluri M., et al. MNRAS (in press, arXiv:1109.3193), (2011) 
Assembling the Puzzle of the Milky Way

[4] Valluri M., Debattista V. P., Quinn T., Moore B., (2010), MNRAS, 403, p. 525

[5] Valluri M., et al., MNRAS (in preparation), (2011)

[6] Stinson G. S., et al., MNRAS, 408, (2010), p. 812

[7] O'Shea B., Derris M., Valluri M., Beers T., Carollo D., ApJ (in preparation), (2011)

[8] Yanny B., et al., AJ, 137, (2009), p. 4377

[9] Carollo D., et al., ApJ, 712, (2010), p. 692 